\title{
We 1010
}

\section{Laboratory Monitoring of $\mathrm{CO} 2$ Migration and Phase Transition Using Complex Electrical Conductivity}

\author{
A. Kirichek* (Delft University of Technology), R. Ghose (Delft University of \\ Technology) \& H.K.J. Heller (Delft University of Technology)
}

\section{SUMMARY}

We explore a new monitoring technique for the $\mathrm{CO} 2$ front propagation and phase transition processes using complex electrical conductivity measurements. A laboratory facility has been built to conduct coreflood experiments under reservoir conditions. $\mathrm{CO} 2$ is injected in both dry and brine-saturated reservoir rock. The objectives of the study are to evaluate and quantify the efficacy of complex electrical conductivity as a monitoring parameter for super-critical CO2 migration in the reservoir rock. Two injection experiments, targeting different injection scenarios, are carried out. In the first experiment, gaseous $\mathrm{CO} 2$ is injected into the core and subsequently the pressure and temperature conditions are gradually altered. The electrical response of the system is measured during the $\mathrm{CO} 2$ phase transition from gaseous to liquid and then to super-critical states. In the second experiment, the CO2 is injected into brinesaturated core while complex electrical conductivity is measured continuously. The experimental data can be adequately described by double Cole-Cole model. The experimental results show a high degree of repeatability and sensitivity. Our observations suggest that complex electrical measurements performed through

borehole logging or cross-hole measurements can be useful for $\mathrm{CO} 2$ storage monitoring. 


\section{Introduction}

The complex electrical conductivity has widely been used as a subsurface monitoring parameter in the last few decades. The applications have been sought in environmental characterization and monitoring (e.g., Pelton et al., 1978) and in interpretation of down-hole measurements in hydrocarbon-bearing rocks (e.g., Vinegar and Waxman, 1984). Several field and laboratory experiments have been conducted to investigate the frequency-dependence of electrical properties in the porous media such as in unconsolidated sands (e.g., Kavian et al., 2012) or in sandstones (e.g., Knight, 1991). Laboratory electrical measurements in the frequency range of $10 \mathrm{kHz}$ to $10 \mathrm{MHz}$ have been performed for saturated rocks.

One of the objectives of the present study is to investigate through laboratory tests if the $\mathrm{CO}_{2}$ phase transition in a porous medium does influence significantly the electrical response at different frequencies. The second important goal is to explore the applicability of complex electrical conductivity in monitoring $\mathrm{CO}_{2}$ migration within a brine-saturated core. To address these questions, a novel experimental facility has been built. The new setup allows us to measure the electrical response of a rock which is partially or fully saturated with $\mathrm{CO}_{2}$, at high temperature and pressure conditions mimicking a deep reservoir. Additionally, the experimental setup allows us to monitor $\mathrm{CO}_{2}$-brine displacements in the porous core. In this abstract, we present our findings regarding the factors that affect the electrical measurements due to $\mathrm{CO}_{2}$ migration and phase transition in the porous sample.

\section{Equipment and measurement technique}

The experimental facility for this study is designed to simulate the conditions of a realistic $\mathrm{CO}_{2}$ storage reservoir. A simplified sketch of the setup is shown in Fig. 1. The centrepiece of the assembly is a cylindrical core of Bentheimer sandstone (26 mm in diameter and $142 \mathrm{~mm}$ in length, 22\% porosity) embedded in a pressure vessel.

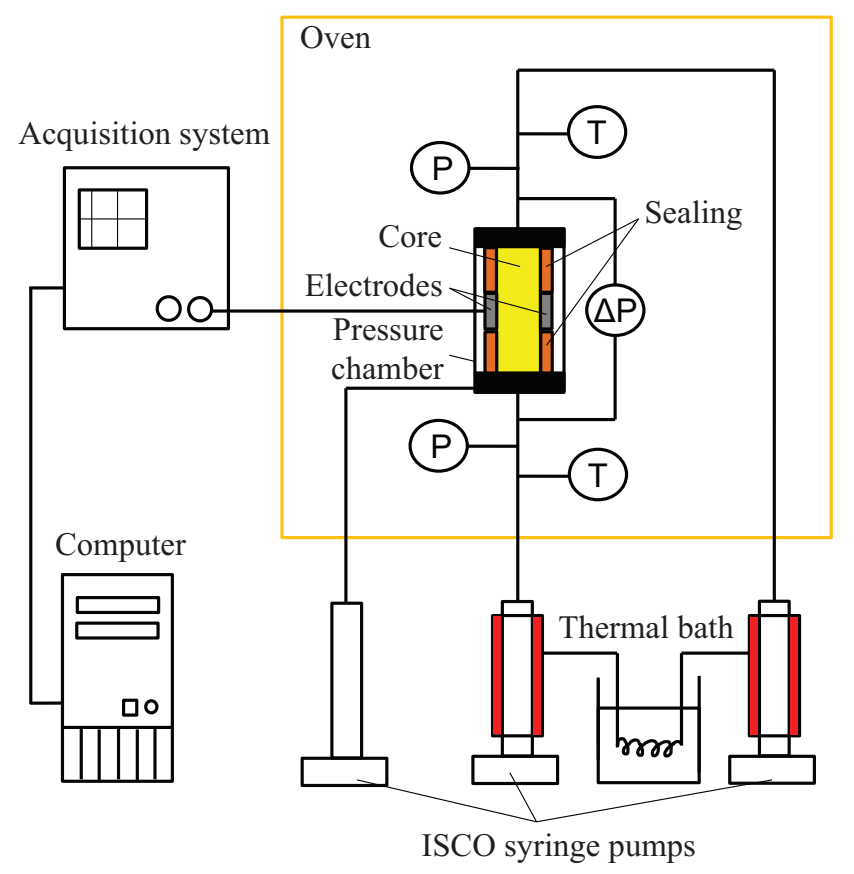

Figure 1 Schematic diagram showing the experimental facility.

Prior to core installation, a pair of electrodes, which measure electrical response of the system, is installed on the lateral surface of the core. The entire surface of the core and electrodes are then sealed using silicone rubber in order to prevent the injection fluid from leaking/infiltrating into the oil used in the hydrostatic pressure chamber. 
The temperature and pressure of the system are controlled by an oven and 3 ISCO syringe pumps, respectively. We employ 2 ISCO syringe pumps, one at each end of the core, for $\mathrm{CO}_{2}$ and brine injection, and one pump generates the confining pressure in the pressure chamber. The pore pressure and the pressure drop over the core are monitored at both ends of the sample. To establish the temperature equilibrium between the core and the injecting fluids, we utilise a thermal bath, which balances the temperature in the oven and in the injection fluids within the pumps. The setup can reproduce reservoir conditions up to 110 bar and maximum temperature up to $65^{\circ} \mathrm{C}$.

Wayne Kerr precision component analyser is used to measure the frequency-dependant amplitude $\left|Z^{*}\right|$ $[\Omega]$ and the phase angle $\phi\left[^{\circ}\right]$ of the complex electrical impedance, which is defined as $Z^{*}=|Z| e^{i \phi}$. Each measurement is conducted at a time interval of 90 second for the frequency range from $20 \mathrm{~Hz}$ to $3 \mathrm{MHz}$.

\section{Phase transition experiment}

The objective of this experiment is to investigate the sensitivity of the complex electrical conductivity to $\mathrm{CO}_{2}$ phase transition. Additionally, we would like to determine the frequency range that is most affected by the $\mathrm{CO}_{2}$ phase change. Initially, the air is removed from the system using a vacuum pump. The empty pores of the core are then filled with $\mathrm{CO}_{2}$ under ambient conditions. In this study, the ambient conditions are defined as $\mathrm{T}=22^{\circ} \mathrm{C}$ and $\mathrm{P}=1$ bar. The experiment is split into two stages: isothermal and isobaric. During the 1st stage, the pore pressure is gradually increased from the ambient condition up to 77.5 bar. The electrical response of the system is continuously measured. During the 2nd stage, the temperature of the system is raised until $52.2^{\circ} \mathrm{C}$, keeping the elevated pressure unchanged.

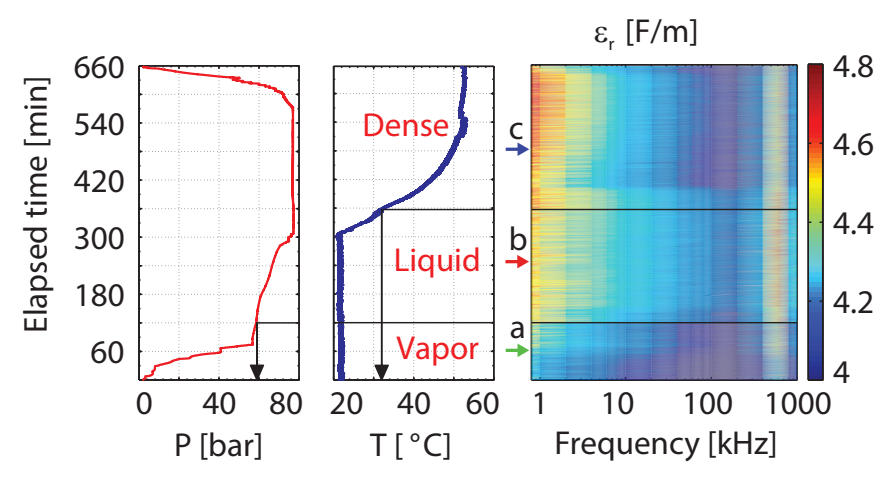

Figure 2 Changes of electrical permittivity during $\mathrm{CO}_{2}$ phase transition. The left and middle panels depict the pressure and temperature evolution over time in the experimental chamber. The right panel shows the electrical permittivity variation over the frequency range from $1 \mathrm{kHz}$ to $1 \mathrm{MHz}$. Black arrows indicate the $\mathrm{CO}_{2}$ critical point.

The component analyser outputs the amplitude $\left|Z^{*}\right|$ and phase $\phi$ data of the complex electrical impedance at different frequencies. For the purpose of $\mathrm{CO}_{2}$ phase transition monitoring, we use only electrical permittivity. Electrical permittivity data is available for different $\mathrm{CO}_{2}$ phases, such as those given by Haynes (2012). The electrical conductivity data is not used because we found limited sensitivity of electrical conductivity to the $\mathrm{CO}_{2}$ phase transition. This is probably due to much greater sensitivity of conductivity to temperature than to phase changes.

The electrical permittivity data is measured over the frequency range from $20 \mathrm{~Hz}$ to $3 \mathrm{MHz}$. We notice that in the frequency range between $800 \mathrm{~Hz}$ and $1 \mathrm{MHz}$ the results are quite stable and there is a systematic difference in electrical conductivity for the three $\mathrm{CO}_{2}$ phases. The repeatability of this observation was confirmed through a number of separate measurements.

The electrical permittivity data measured during $\mathrm{CO}_{2}$ phase transition as a continuous function of time and frequency are shown in Fig. 2. The green (a), red (b) and blue (c) arrows correspond to the electrical 
permittivity for vapour, liquid and dense $\mathrm{CO}_{2}$, respectively. The measured and theoretical data are in a good correspondence.

\section{$\mathrm{CO}_{2}$-brine displacement experiment}

The aim of this experiment is to study the applicability of complex electrical conductivity to monitoring $\mathrm{CO}_{2}$ migration in a storage reservoir. The vapour $\mathrm{CO}_{2}$ is injected into the empty pores of the core. The temperature is set to $\mathrm{T}=38.1{ }^{\circ} \mathrm{C}$. The pressure is then gradually increased to the reservoir condition of 80.7 bar. In order to achieve a gravitationally stable displacement, the brine, as a more dense phase, is injected from the bottom end of the core at a constant flow rate, controlled by a syringe pump. The $\mathrm{CO}_{2}$, due to its lower density, is flooded by another syringe pump from the top end of the core.

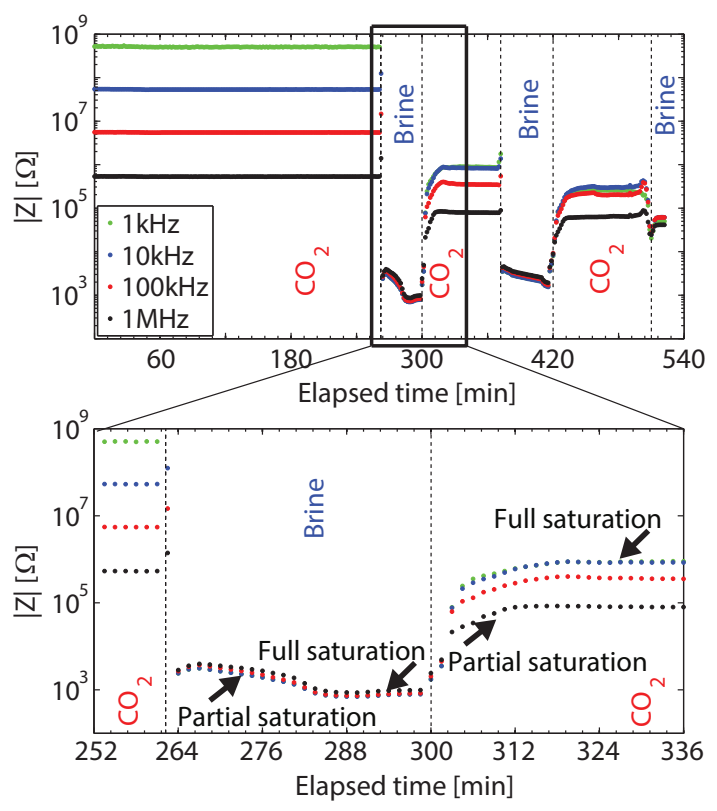

(a)

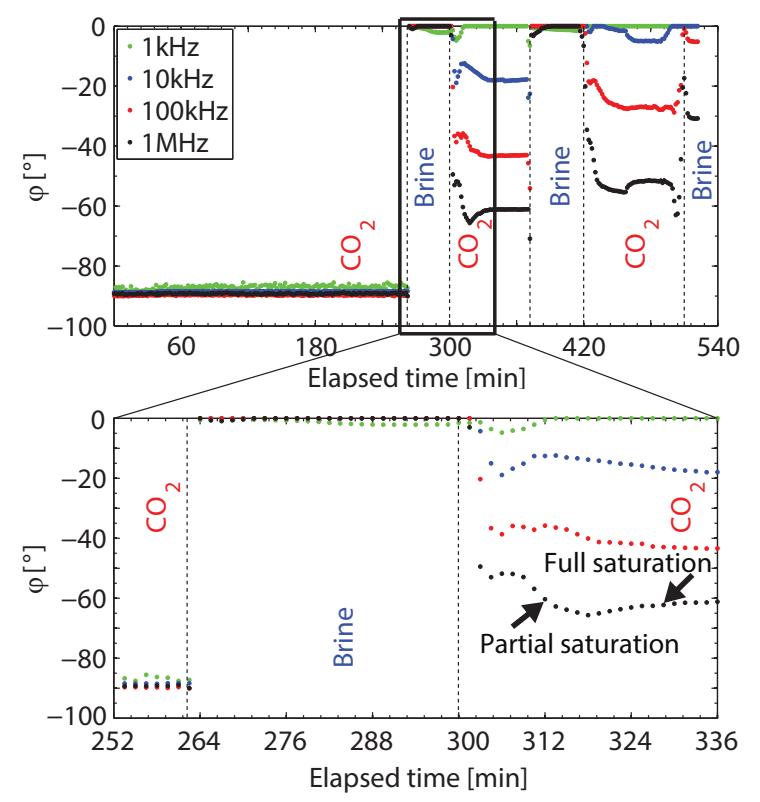

(b)

Figure 3 The changes in amplitude and phase of the complex electrical impedance at $1 \mathrm{kHz}, 10 \mathrm{kHz}$, $100 \mathrm{kHz}$ and $1 \mathrm{MHz}$ during $\mathrm{CO}_{2}$-brine substitution.

The experiment is split into five stages. During the 1st stage, the electrical response of the $\mathrm{CO}_{2}$ saturated system during the pressure build-up is measured. At the 2nd stage, the electrical measurements are conducted during the brine injection into the $\mathrm{CO}_{2}$-filled core. In this study, we use a sodium chloride brine solution with salinity of $10000 \mathrm{ppm}$. The 3rd stage of the experiment involves measurements of the $\mathrm{CO}_{2}$-brine displacement process at a flow rate of $\mathrm{Q}=1 \mathrm{ml} / \mathrm{min}$. The $2 \mathrm{nd}$ and $3 \mathrm{rd}$ stages are repeated to ascertain the repeatability of the results and the sensitivity of the measurements.

The amplitude $\left|Z^{*}\right|$ and phase $\phi$ of complex electrical impedance are shown in Fig. 3 (a) and Fig. 3 (b), respectively. The green, blue, red and black markers represent frequencies $1 \mathrm{kHz}, 10 \mathrm{kHz}, 100 \mathrm{kHz}$ and $1 \mathrm{MHz}$, respectively. The bottom panels focus on the 2 nd and 3rd stages of the experiment when the injected fluid is changed from $\mathrm{CO}_{2}$ to brine and then back to $\mathrm{CO}_{2}$. This corresponds to the elapsed time between 252 and 336 minutes. The black arrows in the bottom panels mark the partial and full saturations of the core.

Both amplitude and phase of the complex electrical impedance demonstrate significant sensitivity to the change of the injected fluid into the core. The partial and full saturation can be clearly distinguished from the amplitude data during the $\mathrm{CO}_{2}$-brine and brine- $\mathrm{CO}_{2}$ substitution. The phase measurements distinguish only the full saturation during the brine- $\mathrm{CO}_{2}$ substitution. However, during the $\mathrm{CO}_{2}$-brine displacement both full and partial saturation effects could be detected from the phase data. 
The experimental results can be adequately described using the empirical Cole-Cole model (Cole and Cole, 1941). For this purpose, one needs DC conductivity, relaxation time, chargeability and the grain size to fit the experimental data in a least square sense. Fig. 4 shows the laboratory measurements and predictions for the partially saturated core using the best-fit parameters.

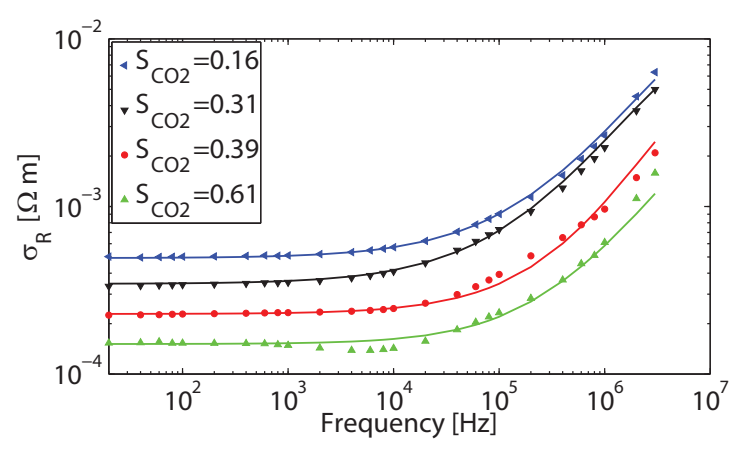

(a)

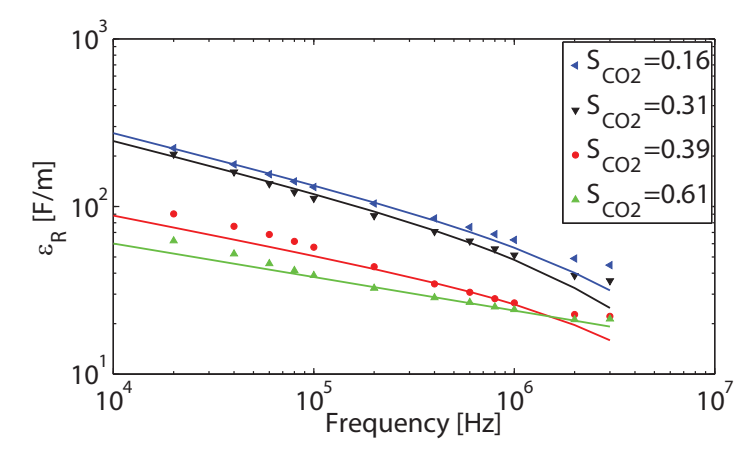

(b)

Figure 4 Measurements of conductivity (a) and permittivity (b) during the partial saturation and the fit with the Cole-Cole model. Small circles represent the measured data when the core is partially saturated with brine and $\mathrm{CO}_{2}$. Solid lines show the predictions using Cole-Cole model.

\section{Conclusions}

One of the objectives of this study was to obtain an insight into the physical processes that occur in the porous media (saturation, phase transition, fluid front movement) and measure the corresponding electrical responses e.g., conductivity and permittivity at different frequencies. The new experiments have allowed us to establish the magnitude range for change of the electrical properties when the porous medium is saturated with either brine or $\mathrm{CO}_{2}$. Our results indicate that complex electrical properties can be used to reduce the uncertainty range in field data interpretation.

The majority of borehole logs focus mostly on the DC conductivity data. The permittivity, which allows to distinguish the specific $\mathrm{CO}_{2}$ phase, is generally neglected. By using the AC electrical measurements, we can acquire and interpret additional down-hole information within a single logging campaign. This can be of special relevance to $\mathrm{CO}_{2}$ storage monitoring projects. The ordinary borehole logs usually have the similar frequency range and physical scale as the experiments we have conducted in the laboratory.

The data of complex electrical conductivity can potentially be acquired in cross-hole measurements and in low-frequency surface surveys. However, this raises the question regarding the scalability of the measurements. A robust approach for upscaling the properties from laboratory to field will be important.

\section{Acknowledgements}

The research has been funded by the Dutch national programme CATO2.

\section{References}

Cole, K. S. and R. H. Cole, (1941), Dispersion and absorption in dielectrics. I. Alternating current characteristics, J. Chem. Phys., 9, 341-351.

Haynes, W. M. (2012), CRC Handbook of Chemistry and Physics, 93rd Edition.

Kavian, M., E. C. Slob and W. A. Mulder (2012), Measured electric responses of unconsolidated layered and brinesaturated sand and sand-clay packs under continuous fluid flow conditions, Journal of Applied Geophysics, 80, 83-90.

Knight, R. J. (1991), Hysteresis in the electrical resistivity of partially saturated sandstones, Geophysics, 56, $2139-2147$.

Pelton, W. H., S. H. Ward, P. G. Hallof, W. R. Sill, and P. H. Nelson (1991), Mineral discrimination and removal of inductive coupling with multifrequency IP, Geophysics, 43, 588-609.

Vinegar, H. J., and M. H. Waxman (1984), Induced polarization of shaly sands, Geophysics, 49 (8), 1267-1287. 